\title{
E1 mantenimiento de los bienes culturales como garantía para su conservación
}

\author{
Concha Cirujano, Ángel Luis García, Teresa Laguna
}

Resumen: La conservación del Patrimonio se debe basar fundamentalmente en la preservación. Cuando se trata de obras expuestas a la intemperie no resulta viable implantar medidas preventivas que permitan controlar las condiciones ambientales que influyen de manera determinante en el desarrollo de los procesos de deterioro. En estos casos, la conservación preventiva se debe centrar en mantener en buen estado los materiales de construcción y los elementos arquitectónicos, tanto estructurales como funcionales o de protección y en la aplicación de programas de mantenimiento. Este tipo de actuaciones permiten la detección precoz de los daños evitando un agravamiento de la patología y son, además, un mecanismo que rentabiliza las inversiones económicas que las instituciones encargadas de la tutela de los bienes culturales dedican a su restauración. Por otra parte, las labores de control y mantenimiento permiten evaluar la idoneidad de los tratamientos y de las soluciones adoptadas.

Palabras clave: Mantenimiento, control, prevención, Sevilla.

Abstract: The Conservation of Cultural Heritage must be based mainly upon preservation. When it comes to in situ outdoor works, it is not possible to apply preventive measures to control the environmental conditions that influence the deterioration process. In these cases, preventive conservation must be focused on maintaining both the structural and functional construction materials and the architectonical elements in a good state, or in protection and also implementation of maintenance programs. These kinds of actions will allow the early detection of damage, avoiding the worsening of the pathology. They will also make profitable the economic investment made for the restorations works by institutions responsible for the protection of cultural heritage. On the other hand, control and maintenance actions allow the assessment of the suitability of the adopted treatments and solutions.

Keywords: maintenance, control, prevention, Sevilla.

Resumo: A conservação do Património deve-se basar fundamentalmente na preservação. Quando se está a tratar de obras expostas à intemperie, não resulta viável implantar medidas preventivas que permitam controlar as condições ambientais que influiem de maneira determinante no desenvolvimento dos processos de deterioração. Nestes casos, a conservação preventiva deve-se centrar em manter em bom estado os materiais de construção e os elementos arquitectónicos, tanto estructurais como funcionais ou de protecção, e na aplicação de programas de manutenção. Este tipo de actuações permitem a deteç̧ão precoz dos danos, evitando um agravamento da patologia e, além disso, são um mecanismo que rentabiliza as inversões económicas que as instituções encarregadas da tutela dos bens culturais dedicam à sua restauração. Por outro lado, as labores de controlo e manutenção permitem avaliar a idoneidade dos tratamentos e das soluções adotadas.

Palavras-chave: Manutenção, controlo, prevenção, Sevilha.

\section{Introducción}

En los últimos años se ha demostrado el beneficio que supone para la conservación de los bienes culturales la aplicación de medidas preventivas que frenen el deterioro, evitando así llegar a situaciones en las que la restauración es la única vía para garantizar la conservación. No solamente existen ventajas desde el punto de vista de la conservación-restauración, sino también desde una 
perspectiva económica, puesto que este tipo de soluciones permite aprovechar de manera más eficaz los recursos que los gobiernos y entidades privadas dedican a este capítulo.

Teniendo en cuenta la incidencia que los factores exógenos tienen en la aparición y desarrollo de los procesos de deterioro de los materiales constitutivos de los bienes culturales, la preservación de los mismos debe fijarse como principal objetivo arbitrar las medidas necesarias para que las condiciones del entorno en el que se encuentran sean las más idóneas, de forma que no se generen nuevos daños o se agraven los ya existentes. Esto es factible cuando hablamos de bienes ubicados en recintos cerrados en los que se puede actuar controlando y, en su caso, modificando las condiciones ambientales. Sin embargo, cuando se trata de bienes expuestos a la intemperie la aplicación de este tipo de medidas es irrealizable.

A los daños producidos por los agentes climáticos, se debe añadir el efecto que la contaminación procedente del tráfico rodado, la industria y las calefacciones tiene sobre la conservación de las superficies de los materiales de construcción. Las naciones firmantes del protocolo de Kyoto se comprometían a reducir para el periodo 2008- 2012 la cantidad de emisiones a la atmósfera en un 5’2\% sobre los niveles de 1990. Para España el compromiso asumido suponía la obligación de no superarlo en más del $15 \%{ }^{1}$. La última reunión del G8, celebrada en el mes de julio en Italia, marcó unos objetivos más ambiciosos a largo plazo que están lejos de conseguirse.

Entre las medidas que se están arbitrando para rebajar las emisiones procedentes del tráfico rodado están aquellas que potencian y facilitan el uso de vehículos no contaminantes o el transporte colectivo, las que gravan el impuesto de matriculación en aquellos vehículos cuyas emisiones superan los 200 gramos de $\mathrm{CO}_{2}$ por kilómetro recorrido ${ }^{2}$, las que promocionan la fabricación y uso de automóviles con combustibles alternativos (nitrógeno, electricidad, gas natural) y las que apuestan por peatonalizar los cascos urbanos.

En cuanto a la reducción de contaminantes procedentes del uso doméstico, el Código Técnico de la Edificación (CTE) ya ha introducido una serie de medidas cuyo objetivo consiste en "...conseguir un uso racional de la energía necesaria para la utilización de los edificios, reduciendo a límites sostenibles su consumo y conseguir asimismo que una parte de este consumo proceda de fuentes de energía renovables como consecuencia de las características de su proyecto, construcción, uso y mantenimiento". No obstante, el CTE se refiere a edificios de nueva construcción y a modificaciones, reformas o rehabilitaciones de edificios existentes con una superficie útil superior a $1.000 \mathrm{~m}^{2}$, en las que se renueve más del $25 \%$ de sus cerramientos, por lo que no afecta a numerosas viviendas que por sus dimensiones no están obligadas a implantar las medidas recogidas en esta Norma. Por tanto, su incidencia en la rebaja de emisiones es hoy por hoy limitada. Por su parte, para la industria existen sistemas que reducen la concentración de óxidos de nitrógeno emitidos, que no están implantados de forma generalizada.

Actualmente, a pesar de la implantación de algunas de estas medidas, las ciudades españolas con una población superior a los 500.000 habitantes superan varias veces al año los valores límites diarios establecidos por la legislación vigente, tanto de partículas en suspensión como de algunos gases contaminantes ${ }^{3}$.

En cualquier caso, no hay que olvidar que, aún cuando se consiga mejorar la calidad del aire de la atmósfera y por tanto las condiciones del entorno del patrimonio urbano, una gran cantidad de los bienes se encuentran en el medio rural y tanto unos como otros seguirán bajo la influencia de los factores climáticos y por tanto la prevención solamente podrá basarse en el control de su estado de conservación y en el mantenimiento continuado. 


\section{Metodología para la definición de un programa de control y mantenimiento}

La legislación española, tanto a nivel estatal como autonómico, y los acuerdos internacionales que nuestro país ha suscrito, reconocen la obligación de los poderes públicos de conservar, restaurar y preservar el patrimonio, pero mientras los capítulos presupuestarios dedicados a la restauración suelen ser elevados, las inversiones en su mantenimiento no lo son tanto, cuando deberían instituirse como prioritarias.

Como ya se ha comentado, para conservar los bienes culturales es necesario en muchos casos recurrir a intervenciones de restauración pero no puede olvidarse que estos procesos llevan aparejados ciertos riesgos. La limpieza de las superficies puede acarrear la aparición de nuevos daños en los materiales, puesto que ningún sistema es absolutamente inocuo. A ello se une el peligro que existe de que la eliminación de la suciedad pueda llevar aparejada la pérdida de información, tanto en lo que se refiere a pátinas, revestimientos o a las huellas de labra.

Por ello, cuando este tipo de actuaciones se hace inevitable debería considerarse únicamente como la primera fase de un programa de conservación. La realidad es que cuando la restauración ha finalizado se presupone que ya se ha alcanzado el objetivo de paralizar el proceso de deterioro y de eliminar los riesgos, por lo que la pervivencia de la obra estaría garantizada, sin tener en cuenta que es indispensable diseñar un plan de seguimiento y mantenimiento continuo que requerirá también de unas inversiones periódicas.

Esta metodología de trabajo está claramente definida en la Carta del Restauro de 1987, que define el mantenimiento como "el conjunto de acciones recurrentes en los programas de intervención, encaminadas a mantener los objetos de interés cultural en condiciones óptimas de integridad y funcionalidad, especialmente después de que bayan sufrido intervenciones excepcionales de conservación y/o restauración". Esto implica que no solamente debe realizarse con posterioridad a una restauración, sino que esta tarea debería aplicarse a todas las obras que integran nuestro patrimonio para preservarlo.

Las labores de prevención a partir del control y mantenimiento periódico de los edificios, exigen revisar no solo el estado de los elementos estructurales y funcionales, sino también el estado de conservación de los materiales para detectar la aparición de nuevos daños y efectuar intervenciones mínimas que permitan conservar todos los elementos en perfectas condiciones. Solo así se evitará llegar a una situación en la que las soluciones deban ser más drásticas.

Es evidente que la complejidad, diversidad y magnitud de nuestro patrimonio pueden hacer inviable la aplicación generalizada de una metodología de trabajo como la que aquí se plantea, entre otras cosas por la limitación de los recursos económicos y humanos de que disponen las administraciones públicas. Pero, en muchos casos convendría reflexionar si es más conveniente efectuar una gran inversión en la restauración de un monumento, que ha de someterse de nuevo a los mismos agentes de deterioro, sin garantizar su mantenimiento posterior, o bien distribuir los presupuestos en pequeñas labores de revisión en un número mayor de edificios, consiguiendo de esa forma controlar su evolución.

No es tarea fácil intentar diseñar una metodología de trabajo que abarque la distinta casuística que podemos encontrar en nuestro patrimonio, pero si se puede trazar un modelo genérico aplicable a cualquier bien, sea cual sea la naturaleza de sus componentes y su estado de conservación.

El primer paso será conocer, en la medida de lo posible, el objeto sobre el que se quiere actuar y el entorno en el que se encuentra, cuantificando los daños, identificando los agentes de alteración y su incidencia. 
Cuando se trata de una obra que ha sido previamente restaurada y de la que se posee un conocimiento más o menos exhaustivo de los materiales constructivos, de su estado de conservación y de los agentes y mecanismos de deterioro, es factible evaluar la mayor o menor incidencia de los diferentes agentes externos y la eficacia y durabilidad de las soluciones adoptadas.

En estos casos, el control y mantenimiento es útil además para evaluar los tratamientos realizados porque uno de los peligros que entraña la restauración es la posibilidad de que los productos aplicados en los diferentes procesos interaccionen con el soporte generando subproductos nocivos, o bien que su comportamiento frente a los agentes externos no sea tan satisfactorio como esperábamos. Por ello, esta labor preventiva impediría reincidir en los mismos errores que hayan podido cometerse durante el diseño y ejecución del proyecto.

Tabla 1. CONTROLES PERIÓDICOS SOBRE EL MONUMENTO

\begin{tabular}{|c|c|c|c|c|c|}
\hline & CONTROL & PERIODICIDAD & $\begin{array}{l}\text { RECURSOS } \\
\text { HUMANOS Y } \\
\text { MATERIALES }\end{array}$ & $\begin{array}{l}\text { PLAZO } \\
\text { ESTIMADO }\end{array}$ & $\begin{array}{l}\text { PRESUPUESTO } \\
\text { ESTIMADO }\end{array}$ \\
\hline AMBIENTALES & $\begin{array}{l}\text { Detección de escorrentías } \\
\text { Cohesión soporte } \\
\text { Revisión grietas } \\
\text { Revisión anclajes } \\
\text { Revisión de juntas } \\
\text { Revisión y localización de focos de } \\
\text { corrosión } \\
\text { Localización y cuantificación de } \\
\text { eflorescencias salinas } \\
\text { Revisión consolidación } \\
\text { Revisión hidrofugación }\end{array}$ & & & & \\
\hline CONTAMINATES & $\begin{array}{l}\text { Revisión y cuantificación de } \\
\text { formación de costras } \\
\text { Cuantificación de deposición de } \\
\text { partículas }\end{array}$ & & & & \\
\hline BIODETERIORO & $\begin{array}{l}\text { Revisión y localización de nidos. } \\
\text { Cuantificación excrementos } \\
\text { Revisión crecimiento de plantas... }\end{array}$ & & & & \\
\hline CONSTRUCTIVOS & $\begin{array}{l}\text { Estabilidad de materiales } \\
\text { Revisión uniones y anclajes } \\
\text { Revisión grietas, fracturas } \\
\text { Revisión baberos cornisas } \\
\text { Bajantes, sumideros y cubiertas }\end{array}$ & & & & \\
\hline ANTROPOGÉNICOS & $\begin{array}{l}\text { Localización y cuantificación de } \\
\text { daños: grafittis, manchas, fracturas }\end{array}$ & & & & \\
\hline \multicolumn{2}{|c|}{$\begin{array}{l}\text { MEMORIA Y RECOGIDA DE DATOS } \\
\text { DEL CONTROL }\end{array}$} & & & & \\
\hline \multicolumn{5}{|c|}{ TOTAL PRESUPUESTO CONTROL } & \\
\hline
\end{tabular}

Cuando la obra no ha tenido un proceso previo de restauración, se debe efectuar una inspección para comprobar su estado y detectar, si así fuera, las incipientes o avanzadas alteraciones y sus causas, así como aquellas zonas que por su vulnerabilidad deben ser vigiladas de forma más exhaustiva. 
Para facilitar el trabajo se elaboraría una tabla en la que se recogerían no solamente el tipo de materiales y sus alteraciones, sino los agentes que las provocan e incluso se puede cuantificar la mayor o menor incidencia de cada uno de ellos. Esta valoración podrá revisarse a la vista de la evolución del bien durante los sucesivos controles periódicos que se establezcan.

Tabla 2. INTERVENCIONES ASOCIADAS

\begin{tabular}{|c|c|c|c|c|}
\hline $\begin{array}{l}\text { CONTROL } \\
\text { ASOCIADO }\end{array}$ & INTERVENCIÓN ASOCIADA & $\begin{array}{l}\text { MEDIOS } \\
\text { AUXILIARES }\end{array}$ & $\begin{array}{l}\text { PLAZO } \\
\text { ESTIMADO }\end{array}$ & $\begin{array}{l}\text { PRESUPUESTO } \\
\text { ESTIMADO }\end{array}$ \\
\hline AMBIENTALES & $\begin{array}{l}\text { Limpieza de polvo depositado } \\
\text { Sellado de grietas } \\
\text { Repaso de juntas de mortero } \\
\text { Hidrofugación }\end{array}$ & & & \\
\hline CONTAMINANTES & Limpieza de polvo depositado & & & \\
\hline BIODETERIORO & $\begin{array}{l}\text { Limpieza y retirada de nidos } \\
\text { Reparación de sistemas de alejamiento de } \\
\text { aves } \\
\text { Aplicación de biocida }\end{array}$ & & & \\
\hline CONSTRUCTIVOS & $\begin{array}{l}\text { Sellado de anclajes } \\
\text { Unión fracturas } \\
\text { Reparación de vías de evacuación de aguas } \\
\text { Limpieza bajantes }\end{array}$ & & & \\
\hline ANTROPOGÉNICOS & $\begin{array}{l}\text { Limpieza manchas y graffitis } \\
\text { Reparación de fracturas }\end{array}$ & & & \\
\hline \multicolumn{2}{|c|}{ RECOGIDA DE DATOS Y REDACCIÓN DE MEMORIA } & & & \\
\hline \multicolumn{4}{|l|}{ TOTAL PRESUPUESTO } & \\
\hline
\end{tabular}

En función de estos datos, se puede elaborar un avance de programa de control, con una estimación de las necesidades y plazos, junto con una valoración presupuestaria que contemple los medios auxiliares y los recursos materiales y humanos que se precisarían. La tabla número 1 puede servir de modelo para reflejar que aspectos deberían tenerse en cuenta al diseñar el control de un edificio, en función de los diferentes factores que pueden influir en su estado de conservación. Esta tabla se puede completar con una ficha individualizada de cada elemento en el que de manera más pormenorizada se recojan todas las incidencias y datos que se consideren reseñables.

De acuerdo con las observaciones, se efectuaría un plan de actuación que contemplaría aquellas acciones tendentes a reparar los posibles daños y que, en función de los mismos, tendrán distinto alcance. Una vez iniciado el plan de mantenimiento, se deberá revisar y actualizar la propuesta inicial.

En la tabla número 2 se muestran las intervenciones de mantenimiento de un edificio o elemento singular, asociadas siempre a las consecuencias que pueden tener cada uno de los agentes de deterioro.

Finalmente, es indispensable recoger y archivar toda la información recabada hasta el inicio del programa de conservación preventiva, así como las observaciones recogidas durante la fase de 
control y las labores realizadas durante las intervenciones de mantenimiento. Para que la evaluación a lo largo de los años sea lo más rigurosa posible, es fundamental unificar la terminología y el método de cuantificación de las diferentes alteraciones detectadas, de modo que exista una uniformidad y continuidad que garantice un seguimiento objetivo.

\section{La Catedral de Sevilla, un modelo a seguir}

Esta metodología se ha aplicado con entera satisfacción en las portadas del Nacimiento, Bautismo, Palos, Campanilla, restauradas por el Ministerio de Cultura, y en la puerta del Perdón, restaurada por la Junta de Andalucía, todas ellas pertenecientes a la Catedral de Sevilla.

Las dos primeras se encuentran situadas en la fachada de poniente y comenzaron a levantarse hacia 1447, finalizando la construcción de la obra pétrea en 1449. Más tarde, en 1468, el escultor Lorenzo de Mercadante concluyó la colocación de la decoración escultórica gótica.

Por su parte, la puerta del Perdón, ubicada en el muro norte de la catedral, fue decorada por Miguel Perin entre 1519 y 1521, y fue este mismo artista renacentista el que inició las figuras de las puertas orientales, Palos y Campanilla, entre los años 1521 y 1523.

Los proyectos redactados por el Ministerio contemplaban un plan de conservación preventiva diseñado para llevarlo a cabo de manera periódica y continuada. El Cabildo de la Catedral, propietario y responsable de la conservación de este templo, acogió favorablemente la iniciativa y desde entonces asume anualmente los gastos que se derivan de su implementación.

Las portadas del Nacimiento y del Bautismo presentaban un avanzado estado de deterioro que amenazaba con la supervivencia de las esculturas, modeladas en barro cocido, originalmente policromadas y que conservan restos diferentes de revestimientos aplicados en distintos momentos a lo largo de su historia

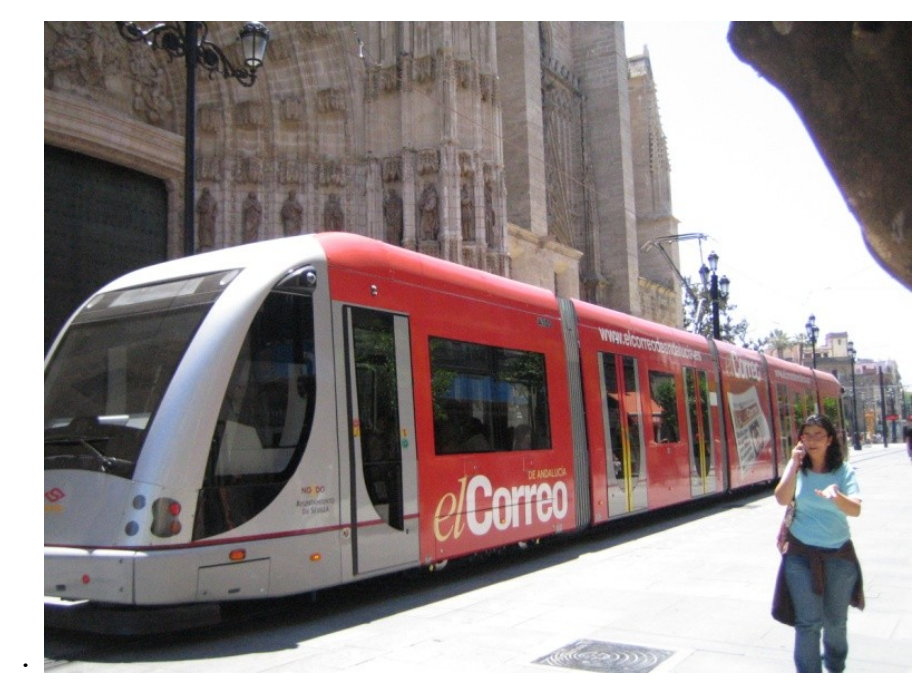

Foto 1.- Avenida en la que se encuentran ubicadas las portadas occidentales, recorrida en la actualidad por un tranvía.

En el año 1992, la Junta de Andalucía 4 , efectuó un estudio exhaustivo de las dos portadas góticas de la fachada occidental, que arrojaron datos sobre las técnicas de ejecución, así como aquellos relativos a los materiales constitutivos, características del medio, factores de alteración y patología. 
Además del acusado deterioro que presentaba la piedra, se comprobó que el principal problema era el avanzado estado de fragmentación y agrietamiento de las figuras de terracota.

La precaria situación de las esculturas motivó que surgieran voces de distintos profesionales que abogaban por retirar los originales al interior del templo, donde estarían perfectamente preservados, colocando en su lugar copias, emulando la solución que ya se había adoptado en otros lugares.

En 1998, el Instituto del Patrimonio Histórico Español inició la intervención de la Portada del Nacimiento y decidió mantener in situ estas piezas, de acuerdo con lo que recoge en su artículo 18 la Ley de Patrimonio Histórico Español 16/855 ya que de acuerdo con el artículo $14^{6}$ de la misma ley, la decoración escultórica tiene la consideración de bien inmueble.

Con el fin de garantizar su conservación, controlar su evolución y evaluar las posibilidades de permanencia en el exterior de las terracotas, se estableció el programa preventivo de control y mantenimiento anteriormente mencionado. No solamente se trataba de incluir en el proyecto un programa de mantenimiento teórico, sino de proponer a largo plazo las acciones necesarias para conservar estas obras y, lo que es más importante, conseguir el compromiso por parte de la propiedad de este bien cultural de llevarlo a cabo.

Este programa comenzó a aplicarse 2 años después de finalizar la restauración de la portada del Nacimiento, coincidiendo con el inicio de la restauración de la portada del Bautismo. Desde entonces hasta junio de 2009 se han ido incorporando sucesivamente el resto de las portadas restauradas por el Ministerio de Cultura, ejecutándose campañas de control y mantenimiento anuales que han sido complementadas desde hace dos años con inspecciones semanales y mensuales.

Los buenos resultados obtenidos fueron el mejor argumento para que los responsables de la conservación de la Catedral de Sevilla decidieran incluir también en este programa la puerta del Perdón, que había sido restaurada por la Junta de Andalucía en 1991 y que desde entonces apenas había sido objeto de labores de conservación por lo que presentaba gruesos depósitos de suciedad y se habían agravado algunas de las grietas existentes en el material cerámico y en los elementos de alabastro, lo que obligó a ponerla previamente a punto mediante una actuación más profunda.

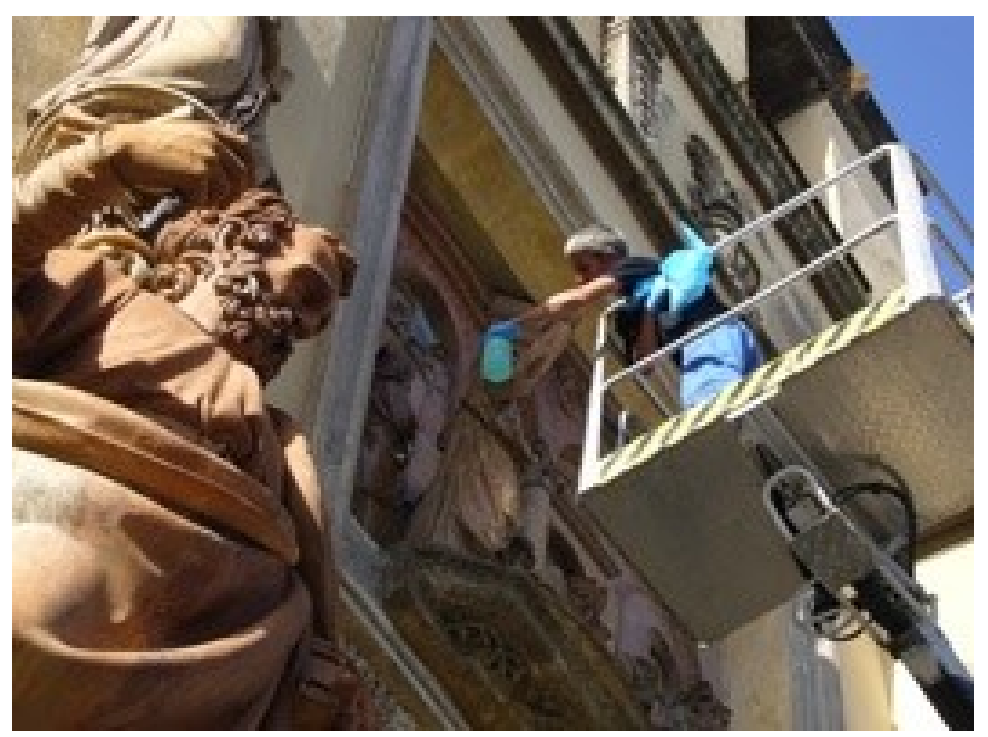

Foto 2- Grúa cesta desde la que se efectúan los trabajos de control y mantenimiento. 
Los primeros trabajos de control y mantenimiento permitieron extraer conclusiones sobre el comportamiento de los diferentes materiales utilizados en la restauración, los cuales se han ido corroborando y ampliando a lo largo de los años.

Gracias a ello se pudo verificar la eficacia del silicato de etilo elegido como consolidante a partir de un estudio realizado sobre probetas de piedra de cantera igual a la original. Se comprobó el buen comportamiento de los morteros de cal y marmolina utilizados para el sellado de grietas. Por el contrario se constató que el teñido en masa de estas lechadas creaba problemas a largo plazo pues se producía un cambio cromático muy acusado. Por ello se optó por entonar los sellados una vez carbonatado el mortero, obteniendo así mejores resultados.

Se desechó el empleo de emulsiones acuosas para fijar las pequeñas exfoliaciones por su rápido envejecimiento provocado por la elevada humedad a que está sometida la ciudad. Se modificaron las dosificaciones de los morteros de rejuntado, se profundizó en los sistemas de limpieza más idóneos y se estudiaron los problemas de funcionamiento que los sistemas de alejamiento de aves generaban; esto último permitió a los proveedores ir perfeccionando los componentes y avanzar en el diseño de las instalaciones.

Pasados 10 años desde la primera intervención, se puede afirmar en líneas generales que tanto el material pétreo como las esculturas de terracota de todas las portadas se encuentran en buen estado, sin que se haya agravado ninguno de los procesos de deterioro que obligaron en su día a una restauración.

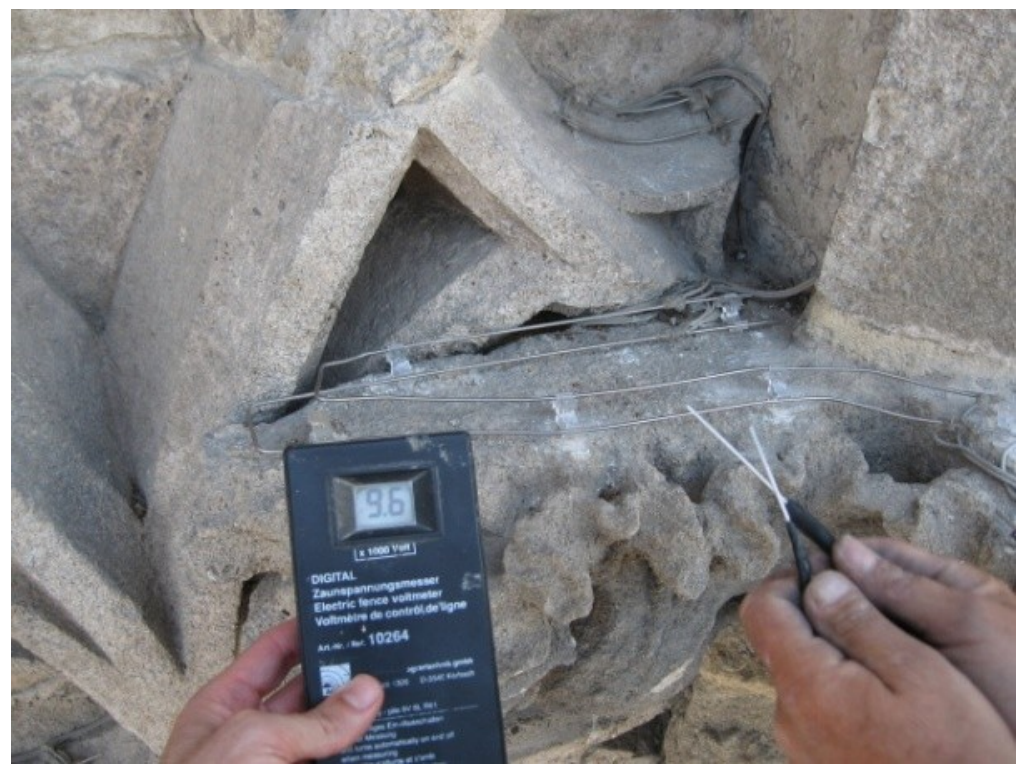

Foto 3- Comprobación del correcto funcionamiento del sistema electrostático en la portada de los Palos.

La piedra de las dos portadas góticas occidentales está más ennegrecida como consecuencia de la contaminación, ya que por la avenida en la que se encuentran transitaba diariamente una gran cantidad de vehículos, fundamentalmente autobuses, lo que unido a la alta humedad relativa provocaba la compactación de las partículas sólidas que se iban depositando sobre la superficie.

Actualmente, la calidad del aire es sensiblemente mejor debido a la eliminación en el año 2007 del tráfico rodado y a la implantación del tranvía como medio de transporte colectivo. La desaparición de uno de los mayores factores de riesgo y, por tanto, la mejora de las condiciones ambientales ha 
redundado en beneficio de todo el monumento y en concreto en estas portadas, como se ha podido comprobar en las dos últimas campañas efectuadas, en las que se ha observado una considerable reducción de los depósitos de suciedad.

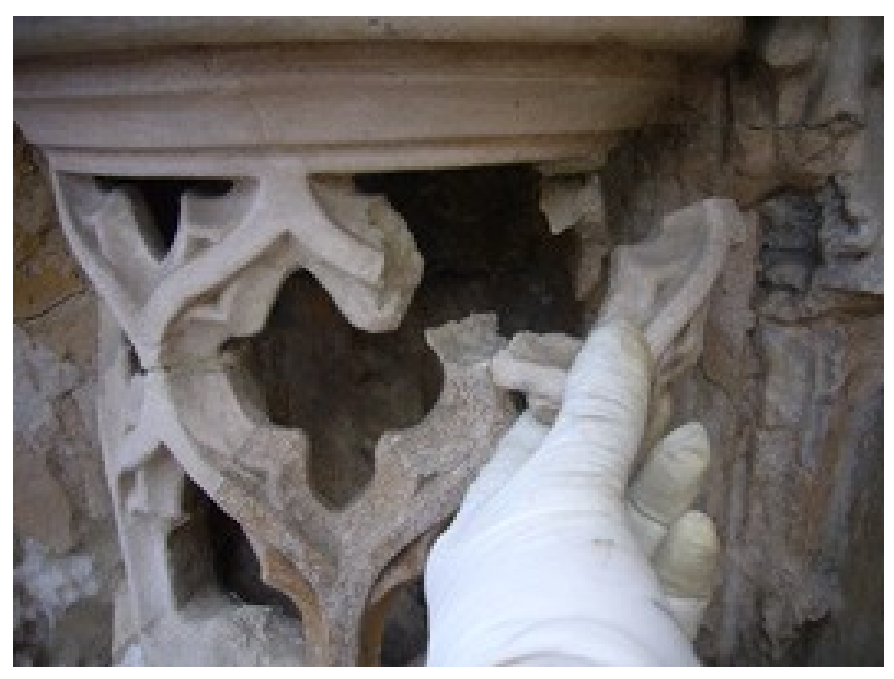

Foto 4.- Detección de una fractura en una pieza de alabastro. Puerta del Perdón.

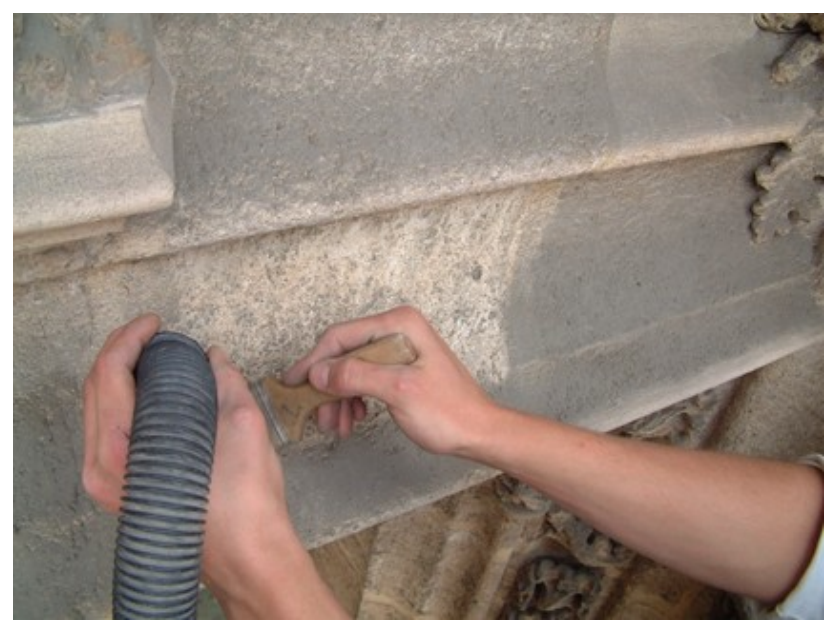

Foto 5. Retirada mediante aspiración de la capa de polvo que recubría las superficies en la portada del Bautismo. Año 2004

A lo largo de este plan de prevención, que ya ha cumplido 10 años, se ha ido precisando la metodología de control haciéndola más eficaz. En cada una de las portadas se han determinado los puntos más vulnerables, seleccionados de acuerdo con los siguientes criterios:

- Elementos que por su geometría son más proclives a la acumulación de suciedad.

- Elementos expuestos a las escorrentías.

- Piezas fracturadas o agrietadas que han sido sometidas a uniones mediante vástagos o a sellados. 
- Sistemas originales de anclaje de las esculturas, en general realizados con metal.

- Zonas de retención de humedad en las que se ha detectado un mayor riesgo de formación de colonias de microorganismos.

- Elementos que por la naturaleza del material (yeso, alabastro, metal) podrían sufrir un mayor deterioro como consecuencia de su exposición a la intemperie.

- Reintegraciones realizadas con materiales que pudieran envejecer bajo el efecto de las radiaciones solares o disgregarse por la acción del agua de lluvia (resinas epoxídicas y morteros)

- En general zonas con patologías que a pesar de las intervenciones realizadas aparecen regularmente como son las grietas por asentamiento del edificio o las producidas por filtraciones desde las terrazas.

Es resumen, todos aquellos elementos o materiales que tanto por su deterioro inicial, por la naturaleza y fragilidad de sus materiales o por su mayor exposición a los agentes externos pueden considerarse más delicados y por tanto deben ser objeto de un seguimiento más exhaustivo.

Las labores de control y mantenimiento se efectúan anualmente, al inicio de la estación estival y durante 5 ó 6 semanas se revisan y se adoptan las soluciones pertinentes. En cuanto a las revisiones semanales y mensuales, el objetivo es recoger gráficamente todas aquellas incidencias que pudieran producirse y se comprueba el buen funcionamiento de los sistemas electrostáticos que se instalaron en las portadas para evitar el anidamiento de las palomas, redactando un pequeño informe que se entrega al Cabildo para su conocimiento y, en su caso, para que adopte las medidas que fueran necesarias.

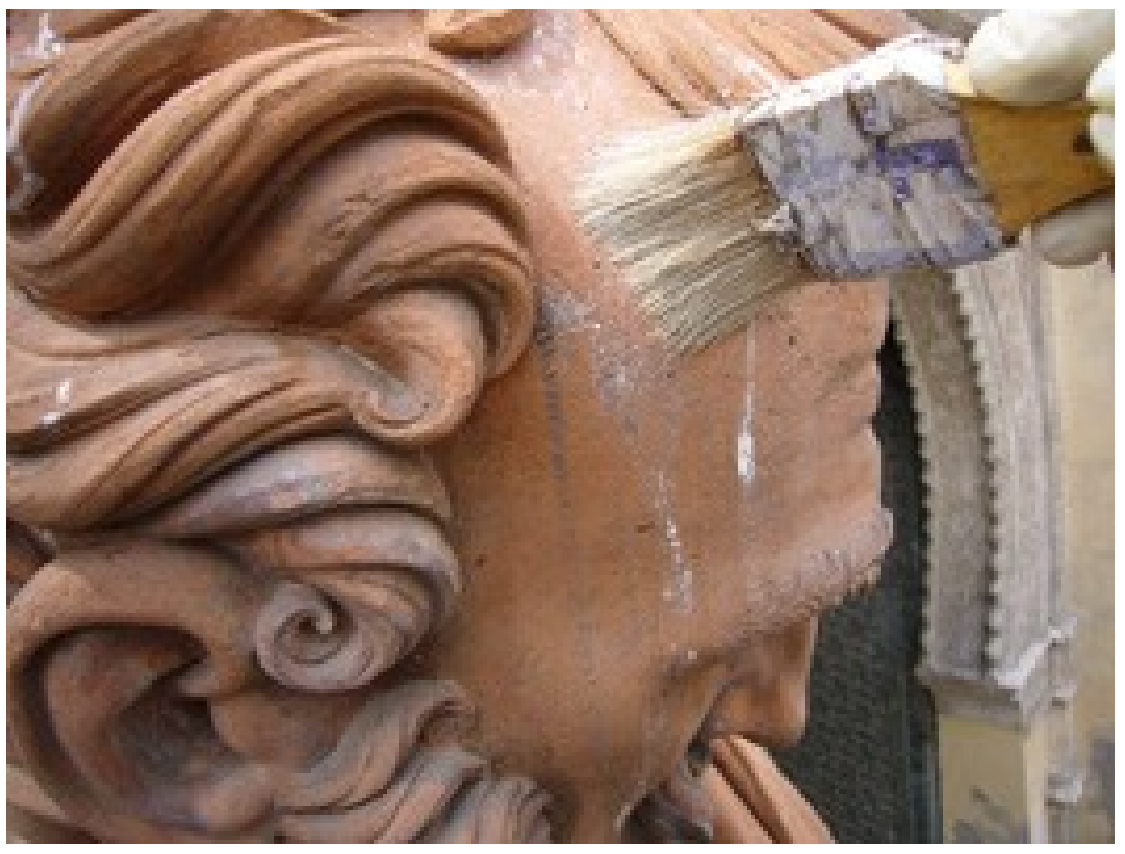

Foto 6- Limpieza de depósitos de guano en la figura de San Pedro de la Puerta del Perdón. Año 2009 


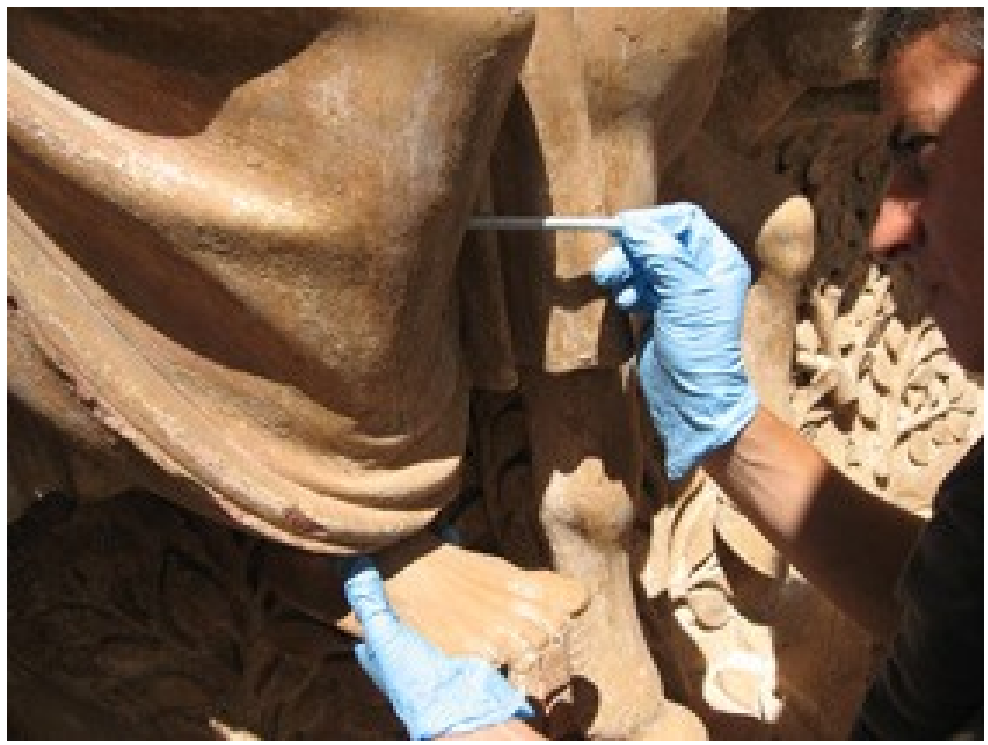

Foto 7- Sustitución de varilla de anclaje en una figura del tímpano de la portada de la Campanilla. Año 2009

Como ya se ha citado, es la propia catedral la que asume los costes económicos de todos los trabajos y facilita una grúa cesta para poder acceder a toda la superficie.

Además de los informes mensuales, se emite uno anual en el que se refleja el estado de conservación durante los controles, las intervenciones de mantenimiento realizadas y todas aquellas sugerencias que se estimen oportunas para la mejor conservación de las portadas. Este documento por tanto sirve de referencia para conocer la evolución de las obras a lo largo de las sucesivas campañas.

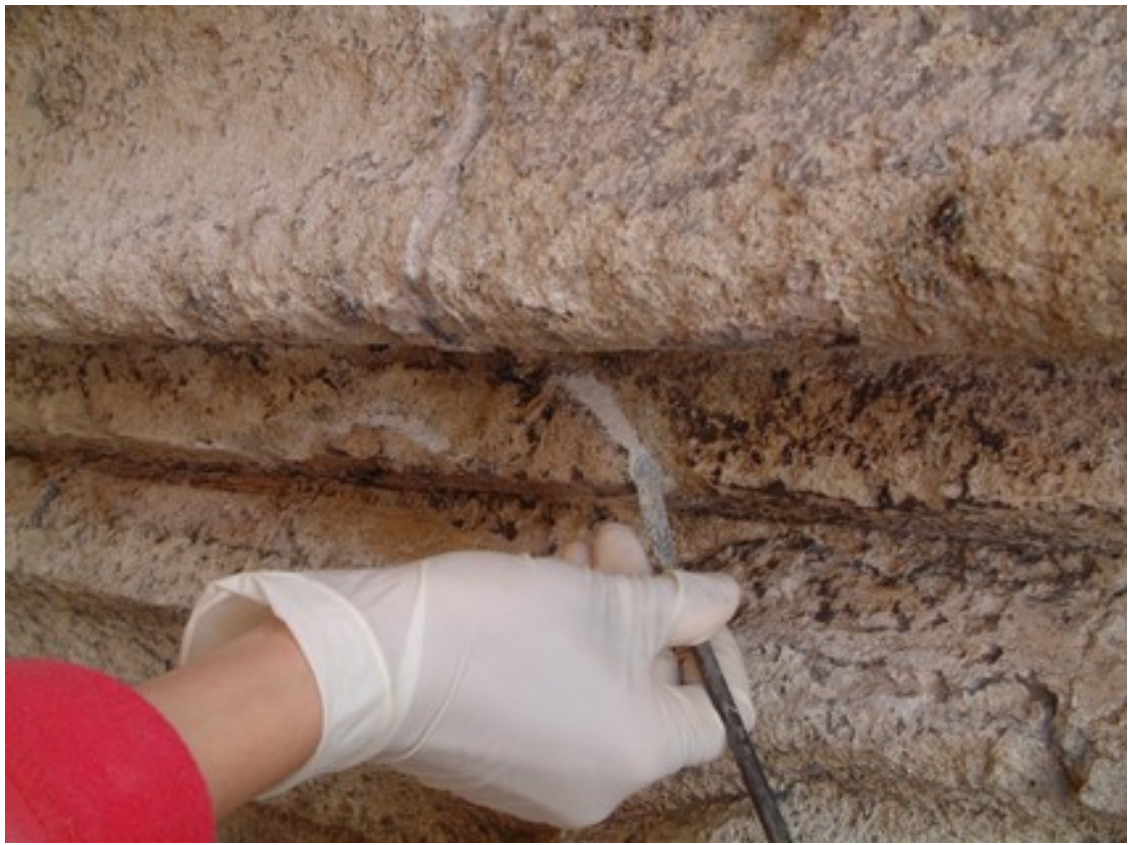

Foto 8.- Sellado de grieta durante los trabajos de mantenimiento en la portada del Nacimiento 
Este ejemplo nos parece que debería ser emulado por otras instituciones que ostentan la propiedad de los bienes o que velan por su conservación, puesto que consideramos que es la mejor forma de asegurar su permanencia.

\section{Agradecimientos}

Al Deán Don Francisco Ortiz Gómez y a Don Francisco Navarro Ruiz, Delegado Ejecutivo de Administración y Patrimonio, por su apoyo y confianza.

A Ana del Castillo, José Antonio Durán Gustavo García, Rosario Giraldez, Carmina Pérez Lázare, Susana Plaza e Isabel Sánchez que han trabajado en condiciones ambientales muy extremas, bajo el sol estival de Sevilla, para llevar a cabo el plan de mantenimiento con una gran profesionalidad y alegría.

\section{Notas}

[1] http://www.icex.es

[2] GÓMEZ, M.V. (6/01/2008). Los impuestos imponen la velocidad. EL PAÍS. NEGOCIOS. $6 / 01 / 2008$

[3] www.ecologistasenaccion.org/IMG/pdf/Modelo plan de acc

[4] PÉREZ RODRÍGUEZ, J L, JIMÉNEZ DE HARO, M C Y JUSTO ERBEZ, A et al. (1993), "Estudio científico de las puertas del Bautismo y Nacimiento de la Catedral de Sevilla". Consejería de Cultura. Junta de Andalucía

[5] LPHE. 16/85. Art. 18.- 1. Un inmueble declarado bien de interés cultural es inseparable de su entorno. No se podrá proceder a su desplazamiento o remoción, salvo que resulte imprescindible por causa de fuerza mayor o de interés socialy, en todo caso, conforme al procedimiento previsto en el artículo 9, párrafo 2 de esta Ley.

[6] LPHE. 16/85. Art. 14.- Para los efectos de esta Ley tienen la consideración de bienes inmuebles, además de los enumerados en el artículo 334 del Código Civil, cuantos elementos puedan considerarse consustanciales con los edificios y formen parte de los mismos o de su entorno o lo hayan formado, aunque en el caso de poder ser separados constituyan un todo perfecto de fácil aplicación a otras construcciones o a usos distintos del suyo original, cualquiera que sea la materia de que estén formados y aunque su separación no perjudique visiblemente al mérito bistórico o artístico del inmueble al que están adheridos.

[7] ARTYCO S.L.

\section{Bibliografía}

CIRUJANO, C. (2002). "Proceso de intervención en las portadas del Nacimiento y del Bautismo de la Catedral de Sevilla". Bienes Culturales. IPHE, 1:101-

CIRUJANO, C.; GUERRA-LIBRERO, F.; LAGUNA PAÚL, T. (2002). "Metodología de intervención en las portadas del Nacimiento y del Bautismo de la Catedral de Sevilla. Programa de mantenimiento". ICOM. 13th Trienal Meeting Rio de Janeiro.

"Memoria de la restauración de la Puerta del Nacimiento de la Catedral de Sevilla". Archivo del Instituto del Patrimonio Cultural de España. 
"Memoria de la restauración de la Puerta del Bautismo de la Catedral de Sevilla". Archivo del Instituto del Patrimonio Cultural de España.

"Memoria de la restauración de la Puerta de los Palos de la Catedral de Sevilla". Archivo del Instituto del Patrimonio Cultural de España.

"Memoria de la restauración de la Puerta de la Campanilla de la Catedral de Sevilla". Archivo del Instituto del Patrimonio Cultural de España.

Carta de 1987 de la Conservación y Restauración de los objetos de arte y cultura.

http://www.mcu.es/patrimonio/docs/MC/PHE/Biblioteca/ITALIA.pdf (consulta: 31/07/09)

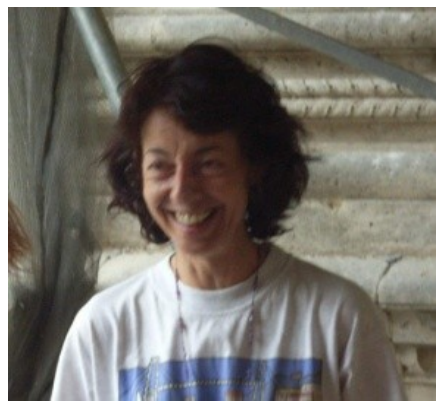

\section{Cirujano Gutiérrez}

Instituto del Patrimonio Cultural de España. Ministerio de Cultura.

Calle Greco no 4. 28040 Madrid

concha.cirujano@mcu.es

Concha Cirujano Gutiérrez Graduada por la Escuela Superior de Conservación de Bienes Culturales. Becada por UNESCO-ICCROM al "8 th Technology of preservation and treatment of stone". Desde 1982 restauradora del Instituto del Patrimonio Cultural de España. Medalla de Oro de Bellas Artes 2007.

\section{Teresa Laguna Paúl}

Catedral de Sevilla. Avenida de la Constitución s/n.41004 Sevilla.

Doctora en Geografía e Historia. Profesora Titular de la Universidad de Sevilla. Desde 1998 desarrolla para la catedral un proyecto de gestión de las colecciones. Premio Nacional de Conservación y Restauración de Bienes Culturales 2002, compartido con el arquitecto Don Alfonso Jiménez Martín.

\section{Ángel Luis García Pérez}

Arte conservación y restauración, S.L. (ÁRTYCO, S.L.). C/ Los Herrán, 44 - $3^{\circ}$ C. 01003 Vitoria Titulado por la Escuela Superior de Conservación de Bienes Culturales. Desde 1989 ha participado en numerosos proyectos, destacando las intervenciones y el mantenimiento de las portadas de la catedral de Sevilla. Desde 2005 forma parte de la sociedad ARTYCO. 
